\title{
Similarity Laws for Stressing Heated Wings
}

\author{
H. S. TSIEN* \\ California Institute of Technology
}

\begin{abstract}
SUMMARY
It will be shown that the differential equations for a heated plate with large temperature gradient and for a similar plate at constant temperature can be made the same by a proper modification of the thickness and the loading for the isothermal plate. This fact leads to the result that the stresses in the heated plate can be calculated from measured strains on the unheated plate by a series of relations, called the "similarity laws." The application of this analog theory to solid wings under aerodynamic heating is discussed in detail. The loading on the unheated analog wing is, however, complicated and involves the novel concept of feedback and "body force" loading. The problem of stressing a heated box-wing structure can be solved by the same analog method and is briefly discussed.
\end{abstract}

\section{INTRODUCTION}

$T$ HE HIGH STAGNation TEMPERATURe for flight of aircraft at supersonic speeds results in severe aerodynamic heating of the surfaces of aircraft. For instance, in a recent paper by Kaye, ${ }^{1}$ the transient temperature distribution in a wedge-shaped solid wing was calculated for accelerated flight and was found to be rapidly varying both with respect to the space points in the wing and with respect to time instants. When there are large temperature gradients in the material, there are generally large thermal stresses due to the uneven thermal expansion of the material. Therefore, concurrent with the severe aerodynamic heating, there is the problem of determining the thermal stresses in the wing. The purpose of this paper is to suggest a method of stressing such heated wings.

The starting point of the stress analysis is the temperature distribution in the wing. Because the rate of change of temperature is small in comparison to the speed of sound propagation in the material, the stress calculation can be considered as a quasi-steady problem. That is, the stress in the wing at each time instant can be calculated from the temperature distribution at that

\footnotetext{
Received June 10, 1952.

* Robert H. Goddard Professor of Jet Propulsion, Daniel and Florence Guggenheim Jet Propulsion Center.
}

time instant without the inertia effects from the time variation of the material displacements required by the changing stresses and the thermal expansions. This problem in general theory of elasticity is greatly simplified by the fact that the wing is thin, and therefore the Kirchhoff hypothesis for bending of thin plates holds. In fact, the problem of thermal stresses in an elastic plate has been treated some time ago by Nádai. ${ }^{2}$ The present study generalizes the earlier theory in two directions: Nádai's assumption of linear temperature profile across the plate is no longer necessary. The temperature profile is now arbitrary. Secondly, Young's modulus $E$ of the material is now allowed to vary as a function of temperature. Thus, the effects of decrease in Young's modulus with increase in temperature can be taken into account in the present theory.

However, the main purpose of the present paper is not just to construct a theory for heated plates. The main purpose is to utilize the theory to formulate a law of similarity, whereby the problem of stressing a heated wing can be solved by performing a set of experiments on a properly proportioned and properly loaded wing at room temperature without heating. This concept of similarity law has been explored before by the present author ${ }^{3}$ for thin-walled cylinders. It is believed that this approach to the problem of stressing a heated wing has many practical advantages over a pure analytical solution, as will be discussed later in the paper.

\section{Basic Equations for Heated Plate}

Let the plane of the plate be the $x-y$ plane, and its variable thickness be $b(x, y)$. The $z$-axis is normal to the plate and pointing downward. The temperature above the ambient is specified by $T(x, y ; z)$. Actually, the distance $z$ is measured from the "median surface," the position of which will be specified presently. Therefore, it is implied here that the median surface, although not exactly coinciding with the $x-y$ plane, is 
nevertheless sufficiently flat to be considered as the $x-y$ plane. Let $u, v$, and $w$ be the displacement of a point $(x, y)$ on the median surface in the $x, y$, and $z$ directions, respectively, due to elastic strain and thermal expansion. Then, according to Kirchhoff's bending hypothesis, the total strains of a point $(x, y, z)$ in the plate are given by

$$
\left.\begin{array}{rl}
\epsilon_{x} & =(\partial u / \partial x)-z\left(\partial^{2} w / \partial x^{2}\right) \\
\epsilon_{y} & =(\partial v / \partial y)-z\left(\partial^{2} w / \partial y^{2}\right) \\
\gamma_{x y} & =(\partial u / \partial y)+(\partial v / \partial x)-2 z\left(\partial^{2} w / \partial x \partial y\right)
\end{array}\right\}
$$

where $\epsilon_{x}$ is the direct strain in $x, \epsilon_{y}$ is the direct strain in $y$, and $\gamma_{x y}$ is the shear strain in the $x-y$ plane. All other strains are small and negligible for thin plates.

Let $E(T)=E(x, y ; z)$ be the variable Young's modulus, $\nu$ the constant Poisson's ratio, and $\sigma_{x}, \sigma_{y}, \tau_{x y}$ the significant components of stresses. Then the strains can be computed from these quantities as follows:

$$
\begin{aligned}
\epsilon_{x} & =\left[\left(\sigma_{x}-\nu \sigma_{y}\right) / E(x, y ; z)\right]+a T(x, y ; z) \\
\epsilon_{y} & =\left[\left(\sigma_{y}-\nu \sigma_{x}\right) / E(x, y ; z)\right]+a T(x, y ; z) \\
\gamma_{x y} & =2(1+\nu) \tau_{x y} / E(x, y ; z)
\end{aligned}
$$

where $a$ is the coefficient of thermal expansion. By solving Eq. (2) for stresses, one has

$$
\left.\begin{array}{rl}
\sigma_{x} & =\frac{E(x, y ; z)}{1-\nu^{2}}\left[\left(\epsilon_{x}+\nu \epsilon_{y}\right)-(1+\nu) a T(x, y ; z)\right] \\
\sigma_{\nu} & =\frac{E(x, y ; z)}{1-\nu^{2}}\left[\left(\epsilon_{y}+\nu \epsilon_{x}\right)-(1+\nu) a T(x, y ; z)\right] \\
\tau_{x y} & =[E(x, y ; z) / 2(1+\nu)] \gamma_{x \nu}
\end{array}\right\}
$$

In the theory of thin plates, the important quantities are not the stresses but are rather the sectional forces and the sectional moments derived from the stresses. These sectional quantities are defined as

$$
\begin{gathered}
N_{x}=\int_{\sigma_{x}} d z, \quad N_{y}=\iint_{d z}, \quad N_{x y}=\mathcal{S}_{\tau_{x y} d z} \\
M_{x}=\int_{\sigma_{x} z d z,} M_{y}=\int_{\sigma_{y} z d z,} M_{x y}= \\
-\mathcal{S}_{\tau_{x y} z d z}
\end{gathered}
$$

where all integrations extend across the whole thickness of the plate. $N_{x}$ and $N_{y}$ are then sectional normal forces, $N_{x y}$ is the sectional shear, $M_{x}$ and $M_{y}$ are the sectional bending moments, and $M_{x y}$ is the sectional twisting moment. By substituting Eq. (1) into Eq. (3) and then into Eqs. (4) and (5), one has, for example,

$$
\begin{aligned}
& N_{x}=D_{0}\left(\frac{\partial u}{\partial x}+\nu \frac{\partial v}{\partial y}\right)-D_{1}\left(\frac{\partial^{2} w}{\partial x^{2}}+\nu \frac{\partial^{2} w}{\partial y^{2}}\right)-N_{T} \\
& M_{x}=D_{1}\left(\frac{\partial u}{\partial x}+\nu \frac{\partial v}{\partial y}\right)-D_{2}\left(\frac{\partial^{2} w}{\partial x^{2}}+\nu \frac{\partial^{2} w}{\partial y^{2}}\right)-M_{T}
\end{aligned}
$$

where

$$
D_{0}(x, y)=\frac{1}{1-\nu^{2}} \int E(x, y ; z) d z
$$

$$
\begin{aligned}
& D_{1}(x, y)=\frac{1}{1-\nu^{2}} \int E(x, y ; z) z d z \\
& D_{2}(x, y)=\frac{1}{1-\nu^{2}} \int E(x, y ; z) z^{2} d z \\
& N_{T} \quad=\frac{a}{1-\nu} \int E(x, y ; z) T(x, y ; z) d z \\
& M_{T} \quad=\frac{a}{1-\nu} \int E(x, y ; z) T(x, y ; z) z d z
\end{aligned}
$$

$N_{T}$ and $M_{T}$ being due to thermal expansion of the material can thus be called thermal sectional normal force and thermal bending moment, respectively. From the above typical expressions for $N_{x}$ and $M_{x}$, it is seen that a simplification results if the median surface is so chosen that

$$
D_{1}=0
$$

In fact, this is the condition for fixing the position of the median surface. With the median surface so determined, the sectional quantities can be calculated as

$$
\left.\begin{array}{c}
N_{x}=D_{0}\left(\frac{\partial u}{\partial x}+\nu \frac{\partial v}{\partial y}\right)-N_{T} \\
N_{y}=D_{0}\left(\frac{\partial v}{\partial y}+\nu \frac{\partial u}{\partial x}\right)-N_{T} \\
N_{x y}=\frac{1-\nu}{2} D_{0}\left(\frac{\partial v}{\partial y}+\frac{\partial u}{\partial y}\right)
\end{array}\right\}
$$

The equilibrium of forces in the medium surface requires

$$
\left.\begin{array}{l}
\left(\partial N_{x} / \partial x\right)+\left(\partial N_{x y} / \partial y\right)=0 \\
\left(\partial N_{x y} / \partial x\right)+\left(\partial N_{y} / \partial y\right)=0
\end{array}\right\}
$$

By substituting Eq. (12) into Eq. (14), the following two equations for $u$ and $v$ are obtained:

$$
\left.\begin{array}{c}
\frac{\partial}{\partial x}\left[D_{0}\left(\frac{\partial u}{\partial x}+\nu \frac{\partial v}{\partial y}\right)\right]+ \\
\frac{1-\nu}{2} \frac{\partial}{\partial y}\left[D_{0}\left(\frac{\partial v}{\partial x}+\frac{\partial u}{\partial y}\right)\right]=\frac{\partial N_{T}}{\partial x} \\
\frac{1-\nu}{2} \frac{\partial}{\partial x}\left[D_{0}\left(\frac{\partial v}{\partial x}+\frac{\partial u}{\partial y}\right)\right]+ \\
\frac{\partial}{\partial y}\left[D_{0}\left(\frac{\partial v}{\partial y}+\nu \frac{\partial u}{\partial x}\right)\right]=\frac{\partial N_{T}}{\partial y}
\end{array}\right\}
$$


When the temperature distribution is specified, the right sides of these equations are known. Then Eq. (15) is a set of simultaneous partial differential equations for $u$ and $v$. If the boundary conditions of the problem are specified in the displacements, Eq. (15) is the proper basis of solution.

If the boundary conditions of the problem are speci- fied in terms of forces instead of displacements, it would be more convenient to use the stress function $\varphi(x, y)$ as the basis of solution. $\Sigma$ is defined through the following relations and satisfies Eq. (14) automatically:

$$
N_{x}=\frac{\partial^{2} \varphi}{\partial y^{2}}, \quad-N_{x y}=\frac{\partial^{2} \varphi}{\partial x \partial y}, \quad N_{\nu}=\frac{\partial^{2} \varphi}{\partial x^{2}}
$$

By substituting these relations into Eq. (12), one has

$$
\left.\begin{array}{rl}
\frac{\partial u}{\partial x}= & \frac{1}{\left(1-\nu^{2}\right) D_{0}}\left[\left(\frac{\partial^{2} \varphi}{\partial y^{2}}-\nu \frac{\partial^{2} \varphi}{\partial x^{2}}\right)+(1-\nu) N_{T}\right] \\
\frac{\partial v}{\partial y}= & \frac{1}{\left(1-\nu^{2}\right) D_{0}}\left[\left(\frac{\partial^{2} \varphi}{\partial x^{2}}-\nu \frac{\partial^{2} \varphi}{\partial y^{2}}\right)+(1-\nu) N_{T}\right] \\
& -\left(\frac{\partial v}{\partial x}+\frac{\partial u}{\partial y}\right)=\frac{2}{(1-\nu) D_{0}} \frac{\partial^{2} \varphi}{\partial x \partial y}
\end{array}\right\}
$$

When the first equation of Eqs. (17) is differentiated twice with respect to $y$, the second equation is differentiated twice with respect to $x$, the third equation is differentiated with respect to $x$ and $y$, and the results are added, a single equation for $\varphi$ is obtained:

$$
\begin{aligned}
\frac{1}{\left(1-\nu^{2}\right)} \frac{\partial^{2}}{\partial x^{2}}\left[\frac{1}{D_{0}}\left(\frac{\partial^{2} \varphi}{\partial x^{2}}-\nu \frac{\partial^{2} \varphi}{\partial y^{2}}\right)\right]+\frac{2}{(1-\nu)} \frac{\partial^{2}}{\partial x \partial y}\left(\frac{1}{D_{0}} \frac{\partial^{2} \varphi}{\partial x \partial y}\right)+ & \\
& \frac{1}{1-\nu^{2}} \frac{\partial^{2}}{\partial y^{2}}\left[\frac{1}{D_{0}}\left(\frac{\partial^{2} \varphi}{\partial y^{2}}-\nu \frac{\partial^{2} \varphi}{\partial x^{2}}\right)\right]+\frac{1}{1+\nu} \nabla^{2}\left(\frac{N_{T}}{D_{0}}\right)=0
\end{aligned}
$$

where $\nabla^{2}$ is the Laplacian operator. At the boundary of the plate (Fig. 1a), if $l, m$ are the direction cosines of the "outside" normal $n$ to the boundary direction $s$, then sectional normal force $N_{n}$ to the boundary and the sectional shearing force $N_{n s}$ along the boundary are given as follows:

$$
\begin{gathered}
N_{n}=\frac{N_{x}+N_{y}}{2}+\frac{N_{x}-N_{y}}{2}\left(l^{2}-m^{2}\right)+N_{x y}(2 l m)=\frac{1}{2} \Delta^{2} \varphi+\frac{1}{2}\left(l^{2}-m^{2}\right)\left(\frac{\partial^{2} \varphi}{\partial y^{2}}-\frac{\partial^{2} \varphi}{\partial x^{2}}\right)-2 l m \frac{\partial^{2} \varphi}{\partial x \partial y} \\
N_{n s}=-\left(N_{x}-N_{y}\right) l m+\left(l^{2}-m^{2}\right) N_{x y}=-\operatorname{lm}\left(\frac{\partial^{2} \varphi}{\partial y^{2}}-\frac{\partial^{2} \varphi}{\partial x^{2}}\right)-\left(l^{2}-m^{2}\right) \frac{\partial^{2} \varphi}{\partial x \partial y}
\end{gathered}
$$

With the boundary described and the boundary forces specified, Eqs. (18), (19), and (20) then give the proper formulation of the problem.

If $p$ is the downward pressure loading on the plate, then the equilibrium of forces normal to the plane of the plate requires

$$
\frac{\partial^{2} M_{x}}{\partial x^{2}}-2 \frac{\partial^{2} M_{x y}}{\partial x \partial y}+\frac{\partial^{2} M_{y}}{\partial y^{2}}=-p-N_{x} \frac{\partial^{2} w}{\partial x^{2}}-2 N_{x y} \frac{\partial^{2} w}{\partial x \partial y}-N_{y} \frac{\partial^{2} w}{\partial y^{2}}
$$

By substituting the sectional moments obtained from Eq. (13) into the above equilibrium equation, an equation for the lateral deflection $w$ results:

$$
\begin{aligned}
\frac{\partial^{2}}{\partial x^{2}}\left[D_{2}\left(\frac{\partial^{2} w}{\partial x^{2}}+\nu \frac{\partial^{2} w}{\partial y^{2}}\right)\right]+2(1-\nu) \frac{\partial^{2}}{\partial x \partial y}\left(D_{2} \frac{\partial^{2} w}{\partial x \partial y}\right)+ & \frac{\partial^{2}}{\partial y^{2}}\left[D_{2}\left(\frac{\partial^{2} w}{\partial y^{2}}+\nu \frac{\partial^{2} w}{\partial x^{2}}\right)\right]= \\
& -\nabla^{2} M_{T}+p+N_{x} \frac{\partial^{2} w}{\partial x^{2}}+2 N_{x y} \frac{\partial^{2} w}{\partial x \partial y}+N_{\nu} \frac{\partial^{2} w}{\partial y^{2}}
\end{aligned}
$$

Part of the boundary conditions will be expressed in terms of $w$ directly. For instance, if the boundary $s$ with outside normal $n$ is a "fixed edge," then on that boundary

$$
w=0, \quad \partial w / \partial n=0
$$


The other part of the boundary.conditions is described in terms of sectional moments. If $l, m$ are the direction cosines of the outside normal, the sectional bending moments $M_{n}, M_{s}$ and the sectional twisting moment $M_{n s}$ on the boundary (Fig. 1b) are, then,

$$
\begin{aligned}
& M_{n}=\frac{M_{x}+M_{y}}{2}+\frac{M_{x}-M_{y}}{2}\left(l^{2}-m^{2}\right)-M_{x y}(2 l m)=-M_{T}-\frac{1+\nu}{2} D_{2} \nabla^{2} w- \\
& \frac{1-\nu}{2} D_{2}\left(l^{2}-m^{2}\right)\left(\frac{\partial^{2} w}{\partial x^{2}}-\frac{\partial^{2} w}{\partial y^{2}}\right)-2(1-\nu) D_{2} l m \frac{\partial^{2} w}{\partial x \partial y} \\
& M_{s}=\frac{M_{x}+M_{\nu}}{2}-\frac{M_{x}-M_{\nu}}{2}\left(l^{2}-m^{2}\right)+M_{x \nu}(2 l m)=-M_{T}-\frac{1+\nu}{2} D_{2} \nabla^{2} w+ \\
& \left.\frac{1-\nu}{2} D_{2}\left(l^{2}-m^{2}\right)\left(\frac{\partial^{2} w}{\partial x^{2}}-\frac{\partial^{2} w}{\partial y^{2}}\right)+2(1-\nu) D_{2} \operatorname{lm} \frac{\partial^{2} w}{\partial x \partial y}\right) \\
& M_{n s}=\left(M_{x}-M_{y}\right) l m+\left(l^{2}-m^{2}\right) M_{x y .}=-(1-\nu) D_{2} l m\left(\frac{\partial^{2} w}{\partial x^{2}}-\frac{\partial^{2} w}{\partial y^{2}}\right)+(1-\nu) D_{2}\left(l^{2}-m^{2}\right) \frac{\partial^{2} w}{\partial x \partial y}
\end{aligned}
$$

For a "free edge," the Kirchhoff boundary conditions are, then,

$$
M_{n}=0, \quad \frac{\partial M_{n}}{\partial n}+\frac{M_{n}-M_{s}}{r}+\frac{\partial M_{n s}}{\partial s}=0
$$

where $r$ is the radius of curvature of the boundary $s$, or

$$
1 / r=(1 / m)(d l / d s)
$$

\section{Plate at Constant Temperature}

For a plate at the constant reference room temperature, all the temperature terms in the equations de-

(a)

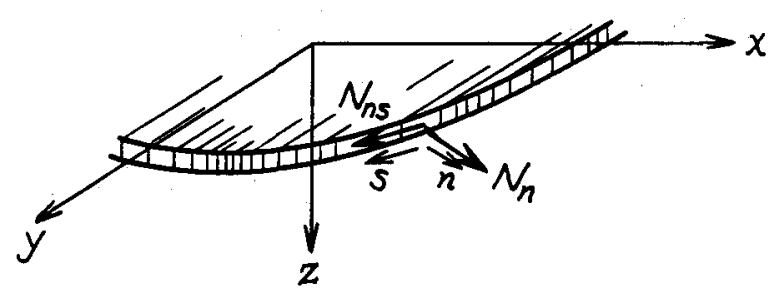

(b)

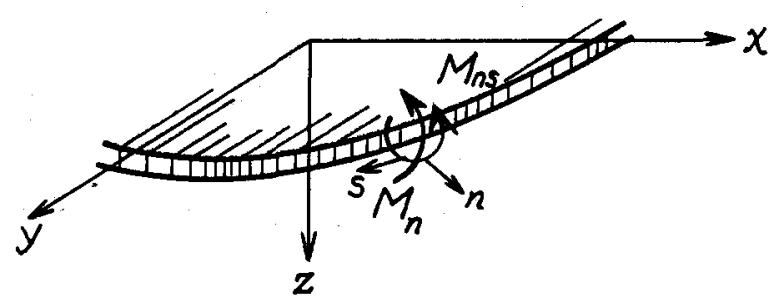

FIG. 1. Boundary forces and moments. veloped in the previous section vanish. By using a bar over a quantity to differentiate the present case from the previous case, one has for strains

$$
\left.\begin{array}{rl}
\bar{\epsilon}_{x} & =(\partial \bar{u} / \partial x)-\bar{z}\left(\partial^{2} \bar{w} / \partial x^{2}\right) \\
\bar{\epsilon}_{y} & =(\partial \bar{v} / \partial y)-\bar{z}\left(\partial^{2} \bar{w} / \partial y^{2}\right) \\
\bar{\gamma}_{x y} & =(\partial \bar{v} / \partial x)+(\partial \bar{u} / \partial y)-2 \bar{z}\left(\partial^{2} \bar{w} / \partial x \partial y\right)
\end{array}\right\}
$$

Let $\vec{E}$ be Young's modulus, now a constant, then

$$
\left.\begin{array}{rl}
\bar{\sigma}_{x} & \left.=\left[\bar{E} /\left(1-\nu^{2}\right)\right]\left(\bar{\epsilon}_{x}+\nu \bar{\epsilon}_{y}\right)\right) \\
\bar{\sigma}_{\nu} & =\left[\bar{E} /\left(1-\nu^{2}\right)\right]\left(\bar{\epsilon}_{y}+\nu \bar{\epsilon}_{x}\right) \\
\bar{\tau}_{x y} & =[\bar{E} / 2(1+\nu)] \bar{\gamma}_{x y}
\end{array}\right\}
$$

The sectional forces and the sectional moments defined as Eqs. (4) and (5) are

$$
\left.\begin{array}{l}
\bar{N}_{x}=\bar{D}_{0}\left(\frac{\partial \bar{u}}{\partial x}+\nu \frac{\partial \bar{v}}{\partial y}\right) \\
\bar{N}_{y}=\bar{D}_{0}\left(\frac{\partial \bar{v}}{\partial y}+\nu \frac{\partial \bar{u}}{\partial x}\right) \\
\bar{N}_{x y}=\frac{1-\nu}{2} \bar{D}_{0}\left(\frac{\partial \bar{v}}{\partial x}+\frac{\partial \bar{u}}{\partial y}\right)
\end{array}\right\}
$$

where, with $\bar{b}(x, y)$ as the thickness of the plate at the point $(x, y)$,

$$
\bar{D}_{0}(x, y)=\frac{\bar{E}}{1-\nu^{2}} \bar{b}(x, y), \quad \bar{D}_{2}(x, y)=\frac{\bar{E}}{1-\nu^{2}} \frac{\bar{b}^{3}}{12}
$$


The median surface now lies midway between the upper and the lower surfaces of the plate.

Now introduce a potential $F(x, y)$ for the body forces $X$ and $Y$, which are the forces per unit area of the median surface in the $x$ and $y$ directions:

$$
X=-\partial F / \partial x, \quad Y=-\partial F / \partial y
$$

Then the equations of equilibrium of forces in the medium surface are

$$
\left.\begin{array}{l}
\left(\partial \bar{N}_{x} / \partial x\right)+\left(\partial \bar{N}_{x y} / \partial y\right)-(\partial F / \partial x)=0 \\
\left(\partial \bar{N}_{x y} / \partial x\right)+\left(\partial \bar{N}_{y} / \partial y\right)-(\partial F / \partial y)=0
\end{array}\right\}
$$

By substituting Eq. (29) into the above equations, a set of equations for $\bar{u}$ and $\bar{v}$ is obtained:

$$
\left.\begin{array}{c}
\frac{\partial}{\partial x}\left[\bar{D}_{0}\left(\frac{\partial \bar{u}}{\partial x}+\nu \frac{\partial \bar{v}}{\partial y}\right)\right]+ \\
\frac{1-\nu}{2} \frac{\partial}{\partial y}\left[\bar{D}_{0}\left(\frac{\partial \bar{v}}{\partial x}+\frac{\partial \bar{u}}{\partial y}\right)\right]=\frac{\partial F}{\partial x} \\
\frac{1-\nu}{2} \frac{\partial}{\partial x}\left[\bar{D}_{0}\left(\frac{\partial \bar{v}}{\partial x}+\frac{\partial \bar{u}}{\partial y}\right)\right]+ \\
\frac{\partial}{\partial y}\left[\bar{D}_{0}\left(\frac{\partial \bar{v}}{\partial y}+\nu \frac{\partial \bar{u}}{\partial x}\right)\right]=\frac{\partial F}{\partial y}
\end{array}\right\}
$$

Eq. (33) will be automatically satisfied, if a new stress function $\bar{\varphi}(x, y)$ is introduced such that

$$
\bar{N}_{x}=\frac{\partial^{2} \bar{\varphi}}{\partial y^{2}}+F, \quad-\bar{N}_{x y}=\frac{\partial^{2} \bar{\varphi}}{\partial x \partial y}, \quad \bar{N}_{y}=\frac{\partial^{2} \bar{\varphi}}{\partial x^{2}}+F
$$

The equation for $\bar{\varphi}$ is now

$\frac{1}{1-\nu^{2}} \frac{\partial^{2}}{\partial x^{2}}\left[\frac{1}{D_{0}}\left(\frac{\partial^{2} \varphi}{\partial x^{2}}-\nu \frac{\partial^{2} \bar{\varphi}}{\partial y^{2}}\right)\right]+\frac{2}{1-\nu} \frac{\partial^{2}}{\partial x \partial y}\left(\frac{1}{D_{0}} \frac{\partial^{2} \bar{\varphi}}{\partial x \partial y}\right)+$

$$
\frac{1}{1-\nu^{2}} \frac{\partial^{2}}{\partial y^{2}}\left[\frac{1}{D_{0}}\left(\frac{\partial^{2} \bar{\varphi}}{\partial y^{2}}-\nu \frac{\partial^{2} \bar{\varphi}}{\partial x^{2}}\right)\right]+\frac{1}{1+\nu} \nabla^{2}\left(\frac{F}{\bar{D}_{0}}\right)=0
$$

At the boundary of the plate,

$$
\begin{aligned}
& \bar{N}_{n}= \frac{\bar{N}_{x}+\bar{N}_{y}}{2}+\frac{\bar{N}_{x}-\bar{N}_{y}}{2}\left(l^{2}-m^{2}\right)+\bar{N}_{x y} 2 l m \\
&=F+\frac{1}{2} \nabla^{2} \bar{\varphi}+\frac{1}{2}\left(l^{2}-m^{2}\right)\left(\frac{\partial^{2} \bar{\varphi}}{\partial y^{2}}-\frac{\partial^{2} \bar{\varphi}}{\partial x^{2}}\right)-2 l m \frac{\partial^{2} \bar{\varphi}}{\partial x \partial y} \\
& \bar{N}_{n s}=-\left(\bar{N}_{x}-\bar{N}_{y}\right) l m+\left(l^{2}-m^{2}\right) \bar{N}_{x y} \\
&=-l m\left(\frac{\partial^{2} \bar{\varphi}}{\partial y^{2}}-\frac{\partial^{2} \bar{\varphi}}{\partial x^{2}}\right)-\left(l^{2}-m^{2}\right) \frac{\partial^{2} \bar{\varphi}}{\partial x \partial y}
\end{aligned}
$$

If $\bar{p}(x, y)$ is the downward pressure loading of the plate at the point $(x, y)$, the equilibrium of lateral forces gives the following equation for $\bar{w}$ :

$$
\begin{aligned}
\frac{\partial^{2}}{\partial x^{2}}\left[\bar{D}_{2}\left(\frac{\partial^{2} \bar{w}}{\partial x^{2}}+\nu \frac{\partial^{2} \bar{w}}{\partial y^{2}}\right)\right]+2(1-\nu) \frac{\partial^{2}}{\partial x \partial y}\left(\bar{D}_{2} \frac{\partial^{2} \bar{w}}{\partial x \partial y}\right)+\frac{\partial^{2}}{\partial y^{2}}\left[\bar{D}_{2}\left(\frac{\partial^{2} \bar{w}}{\partial y^{2}}+\nu \frac{\partial^{2} \bar{w}}{\partial x^{2}}\right)\right]= \\
\bar{p}+\bar{N}_{x} \frac{\partial^{2} \bar{w}}{\partial x^{2}}+2 \bar{N}_{x y} \frac{\partial^{2} \bar{w}}{\partial x \partial y}+\bar{N}_{\nu} \frac{\partial^{2} \bar{w}}{\partial y^{2}}
\end{aligned}
$$

The sectional bending moments $\bar{M}_{n}, \bar{M}_{s}$ and the sectional twisting moment $\bar{M}_{n s}$ are, then,

$$
\left.\begin{array}{l}
\left.\bar{M}_{n}=-\frac{1+\nu}{2} \bar{D}_{2} \nabla^{2} \bar{w}-\frac{1-\nu}{2} \bar{D}_{2}\left(l^{2}-m^{2}\right)\left(\frac{\partial^{2} \bar{w}}{\partial x^{2}}-\frac{\partial^{2} \bar{w}}{\partial y^{2}}\right)-2(1-\nu) \bar{D}_{2} l m \frac{\partial^{2} \bar{w}}{\partial x \partial y}\right) \\
\bar{M}_{s}=-\frac{1+\nu}{2} \bar{D}_{2} \nabla^{2} \bar{w}+\frac{1-\nu}{2} \bar{D}_{2}\left(l^{2}-m^{2}\right)\left(\frac{\partial^{2} \bar{w}}{\partial x^{2}}-\frac{\partial^{2} \bar{w}}{\partial y^{2}}\right)+2(1-\nu) \bar{D}_{2} l m \frac{\partial^{2} \bar{w}}{\partial x \partial y}
\end{array}\right\}
$$



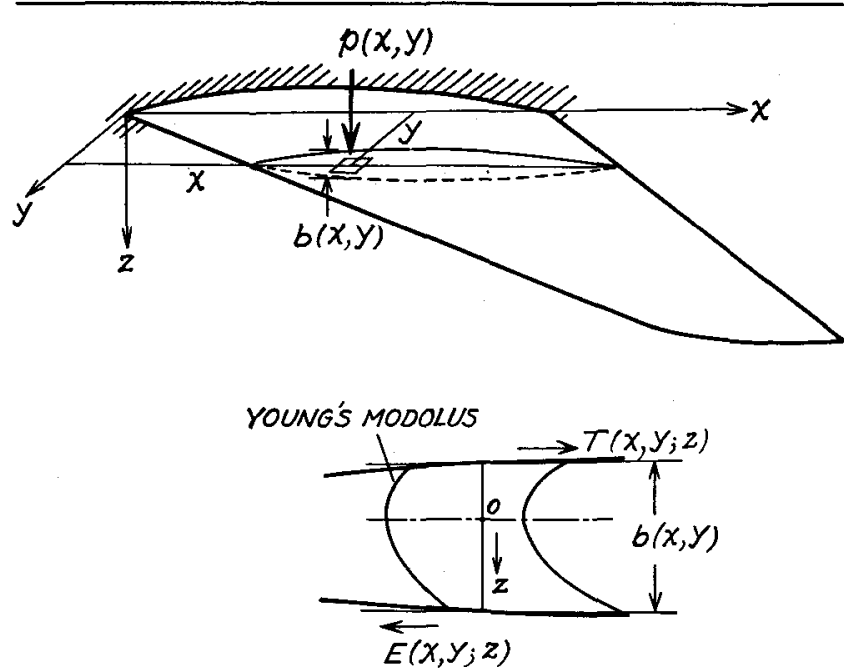

FIG. 2. Loads and boundary conditions of a solid heated wing.

\section{Similarity Laws for Solid ThIN Wings}

By comparing the corresponding equations for the heated plate and for the plate at room temperature, their similarity is evident. The question is then whether it is possible to find a corresponding plate at room temperature such that, with proper loading, the plate will give solutions that are similar to that of the heated plate. To fix ideas, let the heated plate and the unheated plate both have the same plane form. The temperature distribution of the heated plate is specified so that $N_{r}$ and $M_{T}$ are known functions of the coordinates $(x, y)$. The heated plate is built in at one end but free at all other edges (Fig. 2). The lateral pressure loading $p$ for the heated plate is also specified. The problem is to find the thickness distribution $\bar{b}(x, y)$ and the loading of the unheated plate, such that $\bar{\varphi}(x, y)$ and $\bar{w}(x, y)$ are the same as $\varphi(x, y)$ and $w(x, y)$, respectively, except a proportionality factor.

Let $\alpha$ and $\beta$ be two constants; then it is clear that the Eqs. (18) and (36) for $\varphi(x, y)$ and $\bar{\varphi}(x, y)$ will be exactly the same if

$$
\begin{gathered}
\bar{D}_{0}=\alpha D_{0} \\
\bar{\varphi}=\beta \varphi \\
F=\beta N_{T}
\end{gathered}
$$

Then, because of Eqs. (16) and (35),

$$
\begin{array}{ll}
\bar{N}_{x}=\beta\left(N_{x}+N_{T}\right), \quad \bar{N}_{x y}=\beta N_{x y}, & \begin{array}{l}
\bar{N}_{y}= \\
\beta\left(N_{y}+N_{T}\right)
\end{array}
\end{array}
$$

Now, if $\lambda$ and $\mu$ are two additional constants, and

$$
\begin{aligned}
\bar{D}_{2} & =\lambda D_{2} \\
\bar{w} & =\mu w
\end{aligned}
$$

then the equation for $\bar{w}$, Eq. (39), can be rewritten as

$$
\begin{aligned}
& \frac{\partial^{2}}{\partial x^{2}}\left[D_{2}\left(\frac{\partial^{2} w}{\partial x}+\nu \frac{\partial^{2} w}{\partial y^{2}}\right)\right]+2(1-\nu) \frac{\partial^{2}}{\partial x \partial y}\left(D_{2} \frac{\partial^{2} w}{\partial x \partial y}\right)+\frac{\partial^{2}}{\partial y^{2}}\left[\left(\frac{\partial^{2} w}{\partial y^{2}}+\nu \frac{\partial^{2} w}{\partial x^{2}}\right)\right]= \\
& \frac{1}{\lambda \mu} p+\frac{\beta}{\lambda \mu} N_{T} \nabla^{2} \bar{w}+\frac{\beta}{\lambda}\left(N_{x} \frac{\partial^{2} w}{\partial x^{2}}+2 N_{x y} \frac{\partial^{2} w}{\partial x \partial y}+N_{y} \frac{\partial^{2} w}{\partial y^{2}}\right)
\end{aligned}
$$

Therefore it can be deduced, by comparing the above equation with Eq. (21), that, in order for $w$ and $\bar{w}$ to satisfy the same equation,

$$
\begin{gathered}
\lambda=\beta \\
\bar{p}=-\beta N_{T} \nabla^{2} \bar{w}-\beta \mu \nabla^{2} M_{T}+\beta \mu p
\end{gathered}
$$

On the boundary of the heated plate, the forces $N_{n}$ and $N_{n s}$ vanish. By comparing Eqs. (19) and (20) with Eqs. (37) and (38) and by noting Eqs. (43) and (44),

$$
\bar{N}_{n}=\beta N_{T}, \quad \bar{N}_{n s}=0
$$

Similarly, by comparing Eqs. (23) and (24) with Eqs. (37) and (38) and by noting Eqs. (46) and (47), the boundary conditions of Eq. (25) for the free edge of the heated plate reduces to

$$
\left.\begin{array}{c}
\bar{M}_{n}=\beta \mu M_{T} \\
\frac{\partial \bar{M}_{n}}{\partial n}+\frac{\bar{M}_{n}-\bar{M}_{s}}{r}+\frac{\partial \bar{M}_{n s}}{\partial s}=\beta \mu \frac{\partial \bar{M}_{T}}{\partial n}
\end{array}\right\}
$$

The boundary conditions for the built-in edge, Eqs. (22), give

$$
\bar{w}=0, \quad \partial \bar{w} / \partial n=0
$$

As a first application of the theory developed, consider the simple case of a thin wing of solid section: The purpose is to find the stresses in the heated wing by performing experiments on an unheated "corresponding" or analog wing. The first step is to understand clearly the meaning of Eqs. (42) and (46). These equations, in fact, determine the thickness $\vec{b}$ of the unheated wing. Now let $g$ be ratio $E / \bar{E}$ and $\eta$ the nondimensional thickness variable measured from the upper surface of the plate such that $\eta=-1$ at upper surface and $\eta=1$ at the lower surface. Let $\eta_{0}$ be the value of $\eta$ at the median surface. Then,

$$
D_{0}(x, y)=\frac{\bar{E} b}{2\left(1-\nu^{2}\right)} \int_{-1}^{1} g(x, y ; \eta) d \eta
$$




$$
\begin{aligned}
& D_{1}(x, y)=\frac{\bar{E} b^{2}}{4\left(1-\nu^{2}\right)} \int_{-1}^{1} g(x, y ; \eta)\left(\eta-\eta_{0}\right) d \eta \\
& D_{2}(x, y)=\frac{\bar{E} b^{3}}{8\left(1-\nu^{2}\right)} \int_{-1}^{1} g(x, y ; \eta)\left(\eta-\eta_{0}\right)^{2} d \eta
\end{aligned}
$$

By using Eq. (31) and the above relations, the conditions of Eqs. (11), (42), and (46) can be written as

$$
\begin{gathered}
\int_{-1}^{1} g(x, y ; \eta)\left(\eta-\eta_{0}\right) d \eta=0 \\
\frac{\alpha}{2} b(x, y) \int_{-1}^{1} g(x, y ; \eta) d \eta=\bar{b}(x, y) \\
\frac{\lambda}{8} b^{3}(x, y) \int_{-1}^{1} g(x, y ; \eta)\left(\eta-\eta_{0}\right)^{2} d \eta=\frac{1}{12} \bar{b}^{3}(x, y)
\end{gathered}
$$

By eliminating $b, \bar{b}$, and $\eta_{0}$ from these equations, one obtains

$$
\begin{aligned}
\left(\frac{1}{12} \frac{\alpha^{3}}{\lambda}\right)\left(\int_{-1}^{1} g d \eta\right)^{4} & =\left(\int_{-1}^{1} g \cdot \eta^{2} d \eta\right) \times \\
& \left(\int_{-1}^{1} g d \eta\right)-\left(\int_{-1}^{1} g \cdot \eta d \eta\right)^{2}
\end{aligned}
$$

Eq. (56) shows that the "Young's modulus profiles" $g(\eta)$ for various points $(x, y)$ of the heated plate are not entirely arbitrary but must satisfy that relation so that the "similarity" between the heated wing and the isothermal analog wing is possible. If $g$ is a fixed function-i.e., the temperature profiles across the plate are similar-or Young's modulus is constant, then Eq. (56) is certainly satisfied. ${ }^{*}$ For any given problem of a heated wing then, the first step is to compute the value of $\alpha^{3} / \lambda$ or $\alpha^{3} / \beta$ for various points of the wing by Eq. (56). If these values do not differ very much, then, as an approximation, the average of the computed $\alpha^{3} / \beta$ can be used. Then the similarity procedure is possible. But in any event, the value of $\alpha^{3} / \beta$ is fixed by the problem, not at the free choice of the stress analyst. Thus, if $\beta$ is chosen, then $\alpha$ is fixed. Furthermore, $\lambda$ is equal to $\beta$ according to Eq. (48). Therefore, out of the four constants, $\alpha, \beta ; \lambda$, and $\mu$, only two $(\beta$ and $\mu$ ) can be chosen arbitrarily. When this is done, Eq. (54) determines the appropriate thickness $\bar{b}(x, y)$ for the unheated analog wing.

* Mathematically speaking, Eq. (56) is a functional equation. The solution of it gives the explicit required character of $g$. This problem is discussed in the Appendix.
With the geometry of the analog wing determined, the next step is to specify the loads on it. Eq. (50) shows that the unheated wing must be loaded with a sectional tension force $\beta N_{T}$ at the boundary but no sectional shearing force. The first of Eqs. (51) shows that, at the free edge, the analog wing should have a bending moment equal to $\beta \mu M_{T}$. The numerical value of $\mu$ is arbitrary and is at the disposal of the experimenter. The second of Eqs. (51) can be interpreted as an upward support force $-\beta \mu\left(\partial M_{T} / \partial n\right)$ per unit length of boundary but no twisting moment at the boundary. Eq. (52) shows that a built-in edge of the heated wing corresponds to a built-in edge of the analog wing.

On the unheated wing, Eqs. (32) and (44) specify a body force

$$
X=-\beta \frac{\partial N_{T}}{\partial x}, Y=-\beta \frac{\partial N_{T}}{\partial y}
$$

These are forces in the $x$ and $y$ direction per unit area of the wing surface and are loads perhaps new to structures testing. The lateral pressure loading $p$ on the analog wing is specified by Eq. (49). If $\Delta \bar{\epsilon}_{x}$ and $\Delta \tilde{\epsilon}_{y}$ denote the differences of $x$ and $y$ components of direct strains measured on the isothermal wing on the top and the bottom surfaces-i.e., according to Eq. (28)

$$
\left.\begin{array}{l}
\Delta \bar{\epsilon}_{x}(x, y)=\bar{\epsilon}_{x}\left(x, y ;-\frac{\bar{b}}{2}\right)-\bar{\epsilon}_{x}\left(x, y ; \frac{\bar{b}}{2}\right)=\bar{b}(x, y) \frac{\partial^{2} \bar{w}}{\partial x^{2}} \\
\Delta \bar{\epsilon}_{y}(x, y)=\bar{\epsilon}_{y}\left(x, y ;-\frac{\bar{b}}{2}\right)-\bar{\epsilon}_{y}\left(x, y ; \frac{\bar{b}}{2}\right)=\bar{b}(x, y) \frac{\partial^{2} \bar{w}}{\partial y^{2}}
\end{array}\right\}
$$

then Eq. (49) can be written as

$$
\bar{p}=\left(-\beta N_{T} / \bar{b}\right)\left(\Delta \bar{\epsilon}_{x}+\Delta \bar{\epsilon}_{y}\right)-\beta \mu \nabla^{2} M_{T}+\beta \mu p
$$

Needless to say, if there are concentrated lateral loads, the ratio of these loads on the test wing and on the original wing is also equal to $\beta \mu$.

The physical significance of Eq. (59) is different from that of Eq. (57) in that the body force loading is completely specified before the test because $N_{T}$ is a known quantity, while the first term of $\bar{p}$ itself depends upon the experimentally determined $\Delta \overline{\boldsymbol{\epsilon}}_{x}$ and $\Delta \overline{\boldsymbol{\epsilon}}_{y}$. Therefore, if the loads are considered as input to the wing structure and the experimentally measured strains as output, then Eq. (57) shows that the output also partly determines the input. In other words, there is a "feedback link" in the experimental setup of the analog wing.

With the loading on the unheated wing so specified, the relation between $\bar{\varphi}$ and $\varphi$ and between $\bar{w}$ and $w$ are given by Eqs. (43) and (47). The stresses in the original heated wing can then be computed from the measured strains on the test wing. For instance, from Eqs. (1) and (3),

$$
\sigma_{x}(x, y ; z)=\frac{E(x, y ; z)}{1-\nu^{2}}\left[\left(\frac{\partial u}{\partial x}+\nu \frac{\partial u}{\partial y}\right)-z\left(\frac{\partial^{2} w}{\partial x^{2}}+\nu \frac{\partial^{2} w}{\partial y^{2}}\right)-(1+\nu) a T(x, y ; z)\right]
$$


By using Eq. (17), the above equation is reduced to

$$
\sigma_{x}(x, y ; z)=\frac{E(x, y ; z)}{1-\nu^{2}}\left\{\frac{N_{T}}{D_{0}}+\frac{1}{D_{0}} \frac{\partial^{2} \varphi}{\partial y^{2}}-z\left(\frac{\partial^{2} w}{\partial x^{2}}+\nu \frac{\partial^{2} w}{\partial y^{2}}\right)-(1+\nu) a T(x, y ; z)\right\}
$$

But according to Eqs. (42), (43), (29), and (35),

$$
\frac{1}{D_{0}} \frac{\partial^{2} \varphi}{\partial y^{2}}=\frac{\alpha}{\beta} \frac{1}{\bar{D}_{0}} \frac{\partial^{2} \bar{\varphi}}{\partial y^{2}}=\frac{\alpha}{\beta} \frac{1}{\bar{D}_{0}}\left[\bar{N}_{x}-\beta N_{T}\right]=\frac{\alpha}{\beta}\left(\frac{\partial \bar{u}}{\partial x}+\nu \frac{\partial \bar{v}}{\partial y}\right)-\frac{N_{T}}{D_{0}}
$$

However,

$$
\begin{aligned}
& \frac{\partial \bar{u}}{\partial x}=\frac{1}{2}\left[\bar{\epsilon}_{x}\left(x, y ;-\frac{\bar{b}}{2}\right)+\bar{\epsilon}_{x}\left(x, y ; \frac{\bar{b}}{2}\right)\right]=\overline{\bar{\epsilon}}_{x}(x, y) \\
& \frac{\partial \bar{v}}{\partial y}=\frac{1}{2}\left[\bar{\epsilon} y\left(x, y ;-\frac{\bar{b}}{2}\right)+\bar{\epsilon}_{y}\left(x, y ; \frac{\bar{b}}{2}\right)\right]=\overline{\bar{\epsilon}}_{y}(x, y)
\end{aligned}
$$

where $\overline{\bar{\epsilon}}_{x}(x, y)$ is the average of the $x$-strain at the top and the bottom surfaces of the analog wing at the point $(x, y)$ and $\overline{\bar{\epsilon}}_{y}(x, y)$ is the average of the $y$-strain. Thus, together with Eq. (58), the above expressions give

$$
\sigma(x, y ; z)=\frac{E(x, y ; z)}{1-\nu^{2}}\left\{\frac{\alpha}{\beta}\left(\bar{\epsilon}_{x}+\nu \overline{\bar{\epsilon}}_{y}\right)-\frac{z}{\mu \bar{b}}\left(\Delta \bar{\epsilon}_{x}+\nu \Delta \bar{\epsilon}_{y}\right)-(1+\nu) a T(x, y ; z)\right\}
$$

It may be proper to point out again that the quantity $z$ in the above equation and in the subsequent equations is the distance from the median surface, positive if the point is below the median surface. Similar to Eq. (62), one has

$$
\sigma_{\nu}(x, y ; z)=\frac{E(x, y ; z)}{1-\nu^{2}}\left[\frac{\alpha}{\beta}\left(\overline{\bar{\epsilon}}_{\nu}+\nu \overline{\bar{\epsilon}}\right)-\frac{z}{\mu \bar{b}}\left(\Delta \bar{\epsilon}_{\nu}+\nu \Delta \bar{\epsilon}_{x}\right)-(1+v) a T(x, y ; z)\right]
$$

If the difference $\Delta \bar{\gamma}_{x y}$ of the shear strain and the average shear strain $\overline{\bar{\gamma}}_{x y}$ are defined as

$$
\begin{aligned}
& \Delta \bar{\gamma}_{x y}(x, y)=\bar{\gamma}_{x y}\left(x, y ;-\frac{\bar{b}}{2}\right)-\bar{\gamma}_{x y}\left(x, y ; \frac{\bar{b}}{2}\right) \\
& \bar{\gamma}_{x y}(x, y)=\frac{1}{2}\left[\bar{\gamma}_{x y}\left(x, y ;-\frac{\bar{b}}{2}\right)+\bar{\gamma}_{x y}\left(x, y ; \frac{\bar{b}}{2}\right)\right]
\end{aligned}
$$

the shear stress in the original heated wing is calculated as

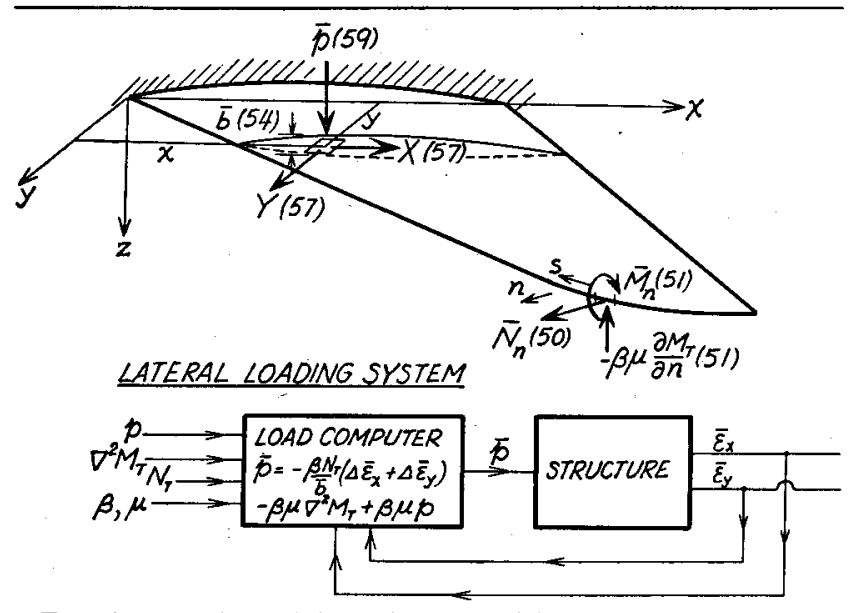

FIg. 3. Loads and boundary conditions of the analog test wing. (Numbers in parentheses correspond to numbered equations in the text where the appropriate relation with quantities of the original heated wing can be found.)

$$
\tau_{x y}(x, y ; z)=\frac{E(x, y ; z)}{2(1+\nu)}\left[\frac{\alpha}{\beta} \overline{\bar{\gamma}}_{x y}(x, y)-\frac{z}{\mu \bar{b}} \Delta \bar{\gamma}_{x y}(x, y)\right]
$$

Eqs. (62), (63), and (66) allow the calculation of the stresses in the heated wing from test data of the unheated wing. They also show the advantage having the constants $(\beta / \alpha)$ and $\mu$ smaller than unity, so that the strains of the unheated wing are magnified in computing the stresses in the heated wing. Then, for a specified load on the heated wing, the test load on the unheated wing is reduced and the analog wing will not be overstrained. Fig. 3 gives a summary of the loading of the unheated test wing, together with the equation numbers of relations that specify the quantities in terms of the quantities of the original heated wing.

\section{Alternate Test Procedure for Thin Solid Wings}

In the previous section, the simulated tests on the isothermal wing are, proposed with simultaneous loading both in the plane of the median surface and in lateral pressure. This results in a rather complicated load system at the free boundary of the wing. To simplify the load system at the boundary, the loading in the plane of the median surface and the lateral loading can be separated-that is, two separate tests, properly correlated, are made on the analog wing, and the stresses in the heated wing are obtained by a synthesis of the test results. 
To determine first the sectional forces $N_{x}, N_{y}$, and $N_{x y}$, the unheated wing is dimensioned to satisfy Eq. (42). It is then loaded with body forces specified by Eq. (57), but no lateral load is applied. At the "free edge" of the wing, it is loaded according to Eq. (50). Then the magnitude of the sectional forces for the heated wing can be computed from Eq. (45) if $\bar{N}_{x}, \bar{N}_{y}$, and $\bar{N}_{x y}$ are determined. These sectional forces can be calculated from the measured strains on the wing. A1though theoretically there should be no bending of the wing, actually bending will be present because of deviations of the real wing from the idealized wing assumed in the theory. The effects of such spurious bending can be eliminated by taking the average of the strains of the top and the bottom surfaces. Let the superscript 1 denote the quantities induced by this loading in the plane of the median surface. Introduce the following notations for the average strains:

$$
\left.\begin{array}{l}
\overline{\bar{\epsilon}}_{x}^{(1)}(x, y)=\frac{1}{2}\left[\bar{\epsilon}_{x}{ }^{(1)}\left(x, y ;-\frac{\bar{b}}{2}\right)+\bar{\epsilon}_{x}^{(1)}\left(x, y ; \frac{\bar{b}}{2}\right)\right] \\
\overline{\bar{\epsilon}}_{y}{ }^{(1)}(x, y)=\frac{1}{2}\left[\bar{\epsilon}_{y}{ }^{(1)}\left(x, y ;-\frac{\bar{b}}{2}\right)+\bar{\epsilon}_{y}{ }^{(1)}\left(x, y ; \frac{\bar{b}}{2}\right)\right] \\
\overline{\bar{\gamma}}_{x y}{ }^{(1)}(x, y)=\frac{1}{2}\left[\bar{\gamma}_{x y}{ }^{(1)}\left(x, y ;-\frac{\bar{b}}{2}\right)+\bar{\gamma}_{x y}{ }^{(1)}\left(x, y ; \frac{\bar{b}}{2}\right)\right]
\end{array}\right\}
$$

Then the sectional forces $N_{x}, N_{y}$, and $N_{x y}$ for the heated wing can be calculated as

$$
\begin{aligned}
& N_{x}(x, y)=\frac{\alpha}{\beta} D_{0}\left[\overline{\bar{\epsilon}}_{x}^{(1)}+\nu \overline{\bar{\epsilon}}_{y}^{(1)}\right]-N_{T} \\
& N_{y}(x, y)=\frac{\alpha}{\beta} D_{0}\left[\overline{\bar{\epsilon}}_{y}^{(1)}+\nu \overline{\bar{\epsilon}}_{x}^{(1)}\right]-N_{T} \\
& N_{x y}=\frac{\alpha}{\beta} \frac{1-\nu}{2} D_{0} \overline{\bar{\gamma}}_{x y}^{(1)}
\end{aligned}
$$

When the average strains of Eq. (67) are measured, the loading in the plane of the median surface, the body forces, and the boundary forces can be removed. The second step of the test is to load the unheated wing laterally. Let Eqs. (46) and (47) be satisfied. Then, in order that $\bar{w}$ and $w$ satisfy the same differential equation, the lateral loading $\bar{p}$ on the test wing must be specified as

$$
\bar{p}=-\lambda \mu \nabla^{2} M_{T}+\lambda \mu p+\lambda\left[N_{x} \frac{\partial^{2} \bar{w}}{\partial x^{2}}+2 N_{x y} \frac{\partial^{2} \bar{w}}{\partial x \partial y}+N_{\nu} \frac{\partial^{2} \bar{w}}{\partial y^{2}}\right]
$$

On the "free edges" of the wing, there is a bending moment equal to $\lambda \mu M_{T}$ and an upward support force $-\lambda \mu\left(\partial M_{T} / \partial n\right)$ per unit length of the boundary.

Let the superscript 2 denote the quantities induced by bending; then,

$$
\left.\begin{array}{l}
\Delta \bar{\epsilon}^{(2)}(x, y)=\bar{\epsilon}_{y}{ }^{(2)}\left(x, y ;-\frac{\bar{b}}{2}\right)-\bar{\epsilon}_{x}{ }^{(2)}\left(x, y ; \frac{\bar{b}}{2}\right)=\bar{b}(x, y) \frac{\partial^{2} \bar{w}}{\partial x^{2}} \\
\Delta \bar{\epsilon}_{y}{ }^{(2)}(x, y)=\bar{\epsilon}_{y}{ }^{(2)}\left(x, y ;-\frac{\bar{b}}{2}\right)-\bar{\epsilon}_{y}{ }^{(2)}\left(x, y ; \frac{\bar{b}}{2}\right)=\bar{b}(x, y) \frac{\partial^{2} \bar{w}}{\partial y^{2}} \\
\Delta \bar{\gamma}_{x y}{ }^{(2)}(x, y)=\bar{\gamma}_{x y}{ }^{(2)}\left(x, y ;-\frac{\bar{b}}{2}\right)-\bar{\gamma}_{x y}{ }^{(22)}\left(x, y ; \frac{\bar{b}}{2}\right)=2 \bar{b}(x, y) \frac{\partial^{2} \bar{w}}{\partial x \partial y}
\end{array}\right\}
$$

With these strains differences between the top and the bottom surfaces, the loading Eq. (69) can be written as

$$
\bar{p}(x, y)=-\lambda \mu \nabla^{2} M_{T}+\lambda \mu p(x, y)+\lambda\left[N_{x} \frac{\Delta \bar{\epsilon}_{x}{ }^{(2)}}{b}+N_{x y} \frac{\Delta \bar{\gamma}_{x y}{ }^{(2)}}{\bar{b}}+N_{y} \frac{\Delta \bar{\epsilon}_{y}{ }^{(2)}}{\bar{b}}\right]
$$

where the sectional forces $N_{x}, N_{y}$, and $N_{x y}$ are computed by using Eq. (68) and the strain data obtained during the first part of the test. This equation again demonstrates the feedback character of the lateral loading. Fig. 4 summarizes the load systems for the two successive tests.

When the strain averages of Eq. (67) and the strain differences of Eq. (70) are measured by the two successive tests on the isothermal analog wing, the stresses in the original heated wing can be computed by using equations similar to Eqs. (62), (63), and (65), replacing $\overline{\bar{\epsilon}}, \overline{\bar{\gamma}}$ by $\overline{\bar{\epsilon}}^{(1)}, \bar{\gamma}^{(1)}$ and $\Delta \bar{\epsilon}, \Delta \bar{\gamma}$ by $\Delta \bar{\epsilon}^{(2)}, \Delta \bar{\gamma}^{(2)}$ of Eqs. (67) and (70), respectively. However, there is one im- portant difference between the test procedure of the present section and the test procedure of the previous one: $\alpha$ can be chosen independent of $\beta$, because now $\beta$ is not necessarily equal to $\lambda$. But $\alpha$ and $\lambda$ are connected because of the condition of Eq. (56). Out of . the four constants $\alpha, \beta, \lambda$, and $\mu$, three can be chosen arbitrarily. This greater degree of freedom and simpler 


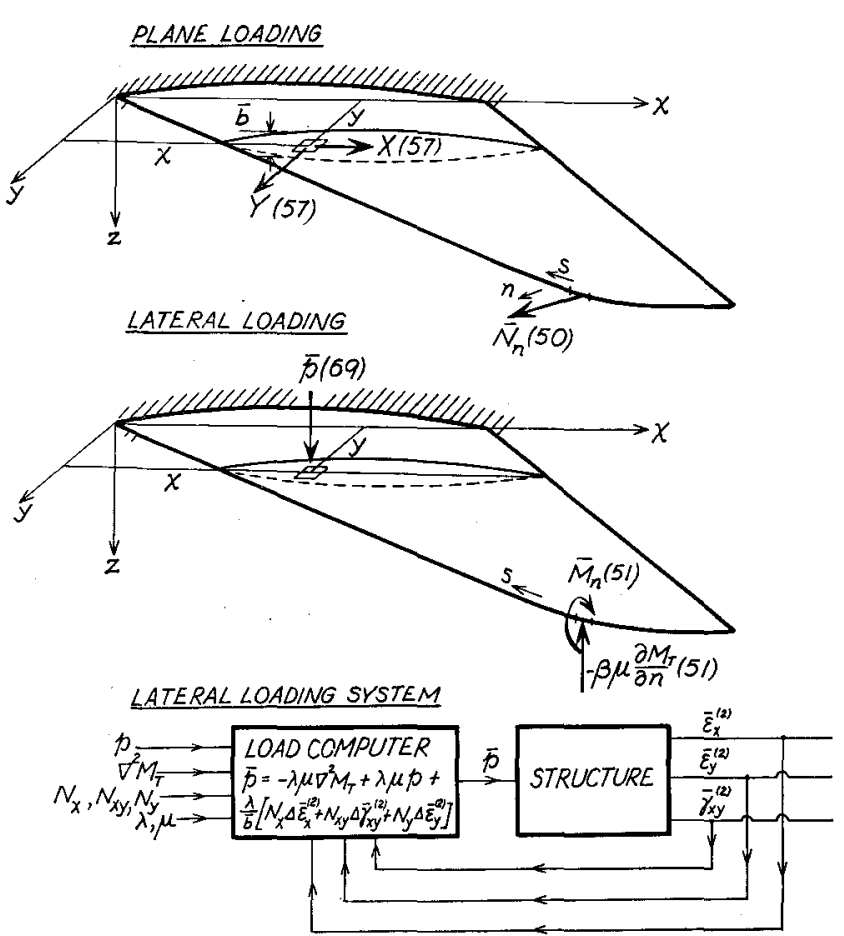

FIG. 4. Loads and boundary conditions of the analog test wing, alternate procedure. (Numbers in parentheses correspond to numbered equations in the text where the appropriate relation with quantities of the original heated wing can be found.)

load system in each stage of tests than the test procedure described in the previous section may prove to be advantageous in actual application.

\section{Box Wing}

In the previous sections, the similarity laws for thin solid wings are formulated for obtaining the stresses in a heated wing by testing an analog unheated wing. A majority of actual wing structures is, however, much more complicated than a solid wing. For example, the main load-carrying member of the wing may be a box structure with top and bottom panels, ribs, and deep beams joining the top and bottom panels. Since only the top and bottom panels of such a box wing are exposed to the air stream, the aerodynamic heating of the structure is limited to these panels.

To analyze such a box wing, the different structure elements can be broken down, and each individual part, such as the top panel, can be considered as a plate. To each individual part then, the theory developed in the earlier sections of this paper can be applied. The thickness $\vec{b}$ for the analog unheated plate for each part of the structure can be specified by the method described previously. The only restriction is that the four constants $\alpha, \beta, \lambda$, and $\mu$ must be the same for all structure elements. The loads on the individual unheated plates corresponding to the different structure elements can be determined according to equations given in the earlier sections. At the boundary or the junction of the different unheated analog panels, there are now two types of load. One type of load comes from the similarity law. This load system is specified previously as the load system for "free edges." The other type of load comes from the junction condition that the deformations of the different parts of the structure must fit together. If the analog panels are put together, then the junction loads will be automatically supplied by the structure. Therefore the procedure to use the method of similarity is as follows:

The first step is to determine the thickness $\vec{b}$ of the analog isothermal box wing by Eqs. (54) and (56). In a box structure, there is appreciable influence on the sectional forces in the median surface of the plates because of bending deflection. Therefore the separation of bending from the "extensional loads" in the plane of the plates is no longer appropriate. The complete load system for the analog wing must be applied simultaneously. This consists of the body force loads in the plane of the plates, the lateral pressure $\bar{p}$, and the "free edge" loads at the junction of the different parts of the structure. The stresses in the original heated box wing can then be calculated by using Eqs. (12), (63), and (65) with the measured strains.

\section{Discussion}

In the previous sections, it is shown that the problem of stressing a heated wing can be solved by tests on an analog unheated wing through the smilarity laws. The temperature distribution in the wing is assumed to be given by a theoretical calculation using aerodynamic heating data and the theory of heat transfer in solid materials. ${ }^{1}$ The loading system required for the analog wing is indeed complicated. Furthermore, the lateral pressure load $\bar{p}$ specified by either $\mathrm{Eq}$. (59) or Eq. (71) involves the concept of feedback in that $\bar{p}$ is partially dependent upon the measured strains and cannot be predetermined. This is, perhaps, a novel concept in structure testing. The possible justification for this great complication in structural testing is the serious difficulty of simulating aerodynamic heating in a structures laboratory and of measuring strains at high temperatures.

The basic principle of the theory of similarity law can be traced to the well-known fact that the general three-dimensional thermal stress problem can be reduced to a problem in isothermal material by introducing appropriate fictitious three-dimensional body forces and surface forces on the boundary of the body. The general three-dimensional formulation of the "analogy" is, however, not useful for structure testing purposes; because there is no possible method of applying three-dimensional body forces. If one dimension of the body is small, such as a thin plate or a thin shell, then the body force is two-dimensional. The loading for the analog isothermal structure can be done, although not easily, as shown in the previous discussion. From this general argument, it is clear then that the present "similarity theory" for thermal stresses can 
certainly be extended to any thin elastic shells, although the actual execution of the analog testing may be more difficult than the flat plate case studied here.

To avoid the task of testing a heated wing, a test wing at room temperature is proposed as an analog. In a sense then, the whole concept of the method of similarity is that of analog-machine computing. There is, however, an advantage of the present method over the recognized machine computer in that the main physical member of the problem, the elastic plate, remains and is not replaced by an approximate system such as electric network. The unheated analog wing is thus the closest analog the aerodynamically heated wing can have and is the most accurate analog. The price of this accuracy and detailed reproduction of the original problem is the complicated test setup required. However, an enterprising structures test engineer will probably welcome such challenge to his ingenuity and demand on his technical skill.

\section{Appendix-Young's Modulus Profile}

By writing $f(\eta)$ as

$$
f(\eta)=\sqrt{(1 / 12)\left(\alpha^{3} / \lambda\right)} g(\eta)
$$

Eq. (56) can be written as

$$
\begin{array}{r}
{\left[\int_{-1}^{1} f(\eta) d \eta\right]^{4}=\left[\int_{-1}^{1} f(\eta) \cdot \eta^{2} d \eta\right]\left[\int_{-1}^{1} f(\eta) d \eta\right]-} \\
{\left[\int_{-1}^{1} f(\eta) \cdot \eta d \eta\right]^{2}}
\end{array}
$$

Now any continuous function $f(\eta)$ for $-1 \leq \eta \leq 1$ can be expanded into a series of Legendre polynomials $P_{n}(\eta)^{*}$-i.e.,

\footnotetext{
* The author is deeply indebted to Prof. A. Erdélyi, of the California Institute of Technology, for suggesting this method of solving the problem.
}

$$
f(\eta)=\sum_{n=0}^{\infty} a_{n} P_{n}(\eta)
$$

where $a_{n}$ 's are constant coefficients. Then ${ }^{4}$

$$
\begin{aligned}
\int_{-1}^{1} f(\eta) d \eta & =\int_{-1}^{1} f(\eta) P_{0}(\eta) d \eta=2 a_{0} \\
\int_{-1}^{1} f(\eta) \eta d \eta & =\int_{-1}^{1} f(\eta) P_{1}(\eta) d \eta=\frac{2}{3} a_{1} \\
\int_{-1}^{1} f(\eta) \eta^{2} d \eta & =\frac{2}{3} \int_{-1}^{1} f(\eta)\left[P_{2}(\eta)+\frac{1}{2} P_{0}(\eta)\right] d \eta= \\
& \frac{2}{3}\left(\frac{2}{5} a_{2}+a_{0}\right)
\end{aligned}
$$

Therefore Eq. (73) can be written as

$$
12 a_{0}^{2}=1+\frac{2}{5}\left(\frac{a_{2}}{a_{0}}\right)-\frac{1}{3}\left(\frac{a_{1}}{a_{0}}\right)^{2}
$$

The restriction of Eq. (73) is thus simply a relation among the first three coefficients $a_{0}, a_{1}$, and $a_{2}$ of the expansion Eq. (74). The later coefficients, $a_{n}, n \geq 3$, are entirely free. Therefore the $f(\eta)$, although not entirely arbitrary, has a wide degree of freedom. Hence, it is probable that the Young's modulus profile will conform to the condition of Eq. (56) for the similarity theory up to a high order.

\section{REFERENCES}

${ }^{1}$ Kaye, J., The Transient Temperature Distribution in a Wing Flying at Supersonic Speeds, Journal of the Aeronautical Sciences, Vol. 17, No. 12, pp. 787-807, 816, December, 1950.

2 Nádai, A., Elastische Platten, p. 264; Julius Springer, Berlin, 1925.

${ }^{3}$ Tsien, H. S., and Cheng, C. M., A Similarity Law for Stressing Rapidly Heated Thin-Walled Cylinders, Journal of the American Rocket Society, Vol. 22, p. 144, 1952.

'See, for instance, Whittaker, E. T., and Watson, G. N., Modern Analysis, Chapt. 15; Cambridge University Press, London, England, 1920. 